\title{
The time course of configural change detection for novel 3-D objects
}

\author{
Simone Favelle and Stephen Palmisano \\ University of Wollongong, Wollongong, New South Wales, Australia
}

\begin{abstract}
The present study investigated the time course of visual information processing that is responsible for successful object change detection involving the configuration and shape of 3-D novel object parts. Using a one-shot change detection task, we manipulated stimulus and interstimulus mask durations (40-500 msec). Experiments $1 \mathrm{~A}$ and $1 \mathrm{~B}$ showed no change detection advantage for configuration at very short $(40-\mathrm{msec})$ stimulus durations, but the configural advantage did emerge with durations between 80 and $160 \mathrm{msec}$. In Experiment 2, we showed that, at shorter stimulus durations, the number of parts changing was the best predictor of change detection performance. Finally, in Experiment 3, with a stimulus duration of $160 \mathrm{msec}$, configuration change detection was found to be highly accurate for each of the mask durations tested, suggesting a fast processing speed for this kind of change information. However, switch and shape change detection reached peak levels of accuracy only when mask durations were increased to 160 and $320 \mathrm{msec}$, respectively. We conclude that, with very short stimulus exposures, successful object change detection depends primarily on quantitative measures of change. However, with longer stimulus exposures, the qualitative nature of the change becomes progressively more important, resulting in the well-known configural advantage for change detection.
\end{abstract}

Observers are often "blind" to large changes to a scene when these changes occur simultaneously with a brief disruption-a phenomenon referred to as change blindness (see Simons, 2000, and Simons \& Levin, 1997, for reviews). Although there are limits to the conclusions that may be drawn from change blindness studies (Simons \& Rensink, 2005), their results do provide important insights into the kinds of visual information that underlie change detection. Spatial layout is one type of information that is likely to be retained in scene representations to support successful change detection. It refers to the overall positioning or placement of the items or elements within a scene/image and does not rely on semantics or the identity of those elements (Hochberg, 1968; Pomerantz, 1983). The retention and representation of spatial layout information is well supported by change detection research with scenes (Aginsky \& Tarr, 2000; Hollingworth \& Henderson, 2002; Rensink, 2000a; Simons, 1996). Simons (1996) investigated change detection for scene displays consisting of multiple objects (both novel and common objects). He found that changes to the spatial layout or configuration of the display were better detected than changes involving either the switching of objects or the replacement of one of the objects. He concluded that the information about the spatial configuration of the scene is easily encoded and represented visually, whereas objectspecific information is not.

Recent change detection research suggests that spatial layout or configural information is also important in the processing of single, complex 3-D objects, as well as of multiobject scenes (Favelle, Hayward, Burke, \& Palmisano, 2006; Favelle, Palmisano, Burke, \& Hayward, 2006; Keane, Hayward, \& Burke, 2003). Configural information, or configuration, is used here to refer to the spatial layout of an object's parts (i.e., the gross part structure of an object). For example, the configuration of one of the objects used in the present study (see Figure 1) could be described as a central body part with three smaller parts, one each attached at the top left, bottom middle, and top right of the body. This is consistent with the idea that the visual system bases object representation on a "part skeleton" that emphasizes structural properties (e.g., the number, location, and spatial relations of parts) over metric properties (Barenholtz, Cohen, Feldman, \& Singh, 2003; Blum, 1973; Kimia, Tannenbaum, \& Zucker, 1995). Note that the shape of the parts is not included in a description of configural information. The configuration does not depend on the parts' being triangular or cigar shaped, nor does it depend on a triangular part's being in a certain location. That is, neither a change to the shape of a part nor a switching of the shape of parts should alter an object's configuration.

Keane et al. (2003) compared the detection of changes made to the configuration of single, novel, complex 3-D objects with changes to the shape of these object's parts. Using a one-shot change detection task, they found that changes to the configuration of the object's parts were

S. Favelle, simone_favelle@uow.edu.au 


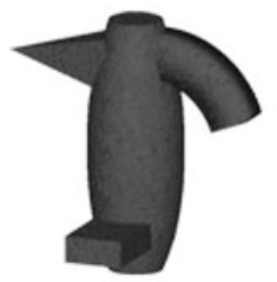

Original

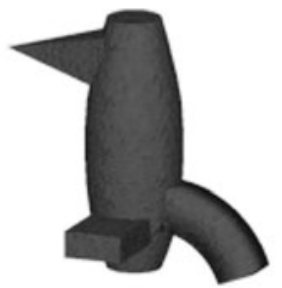

Configural change

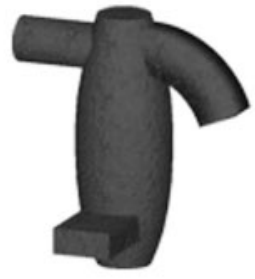

Shape change

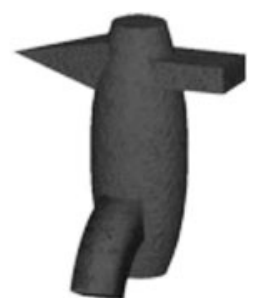

Switch change

Figure 1. Example of the three different types of change (configuration, shape, and switch) used in Experiments 1 and 2 .

more easily detected than changes to the shape of one of those parts or changes involving a switching of the shape of two different parts. In subsequent control experiments, they showed that (1) simple differences in the number of pixels changing could not explain the detection differences observed following configural, switch, and part shape changes; and (2) increasing the size of the object parts (relative to the body) did not negate the configural advantage. A later study by Favelle, Hayward, et al. (2006) found that configural changes to novel 3-D objects were detected more quickly and accurately than part shape or switch changes, regardless of their orientation in depth. Again, an analysis of quantitative measures of the magnitude of change (pixels and color) could not explain differences in performance accuracy between change types. Together, these results imply that the differences in performance between conditions are based primarily on the qualitative nature of the changes. In addition, using visual search and cuing tasks, Favelle, Palmisano, et al. (2006) found that configural changes in 3 -D objects did not attract attention. It was only once the object was attended to that the processing of configural information appeared to be more accurate and faster than the processing of local part shape information.

Configural information has been shown to be important for the perception and recognition of relatively complex 3-D objects, as well as of multiobject scenes. Although speed is just one aspect of efficient information processing, little is known about the temporal characteristics of the extraction and utilization of this configural information. The primary aim of the present article is to address this point. Research has previously examined the time course of configural processing in simple 2-D figures. For example, Kimchi (2000) used a primed matching paradigm to investigate the time course of perceptual organization of simple configurations (line drawings of crosses, squares, and diamonds) and the role of uniform connectedness in this organization. Participants had to make a same-different judgment to a pair of line drawings after they were primed with either connected or unconnected line drawings or control primes. Kimchi (2000) showed that regardless of the connectedness of the prime, and even with prime durations of $40 \mathrm{msec}$, the reaction time (RT) to targets with similar configurations was faster than that to targets with similar components. That is, there is early configural representation of both connected and disconnected line segments. Kimchi's $(2000,2003)$ results suggest that there is an explicit representation of the spatial layout of simple 2-D features, such as oriented lines, in early vision. But the question remains as to the time course of processing configural information in more complex or ecological visual stimuli (e.g., 3-D objects). In addition to determining whether configural information enjoys the same early processing advantage in $3-\mathrm{D}$ as in $2-\mathrm{D}$ object recognition, the use of 3-D stimuli in the present experiment means that the results are relevant to the debate on theories of object recognition, which typically centers on the importance of 3-D structure.

The present study investigated the time course of configural information processing involved in a relatively simple 3-D object change detection task. If we assume that the internal representation of a visual stimulus develops over time, then only an early representation of the stimulus would be available for use in such a change detection task at very short stimulus durations (Kimchi, 2003). Consequently, detecting changes to information that is available in these early representations of the stimulus should be facilitated. Richer stimulus representations would become available at longer stimulus durations, in which case, change detection should also be facilitated for the other types of information available in these representations. Thus, varying the stimulus durations and the type of information changing in a change detection task should reveal the information available in earlier and later representations of the visual stimuli.

In three experiments, we investigated the detection of configural and part shape changes using a one-shot change detection task (as in Keane et al., 2003). Stimulus presentation times for our 3-D objects and interstimulus mask durations were varied so that the processes of information extraction and encoding might be interrupted at different stages in their progress. Specifically, in Experiments 1A and 1B, we varied the stimulus duration $(40-500 \mathrm{msec})$ in order to determine the minimum stimulus exposure required to extract configural object information for successful change detection. In Experiment 2, we compared configural change detection to a quantitative measure of change in terms of the number of parts changing. In Experiment 3, we varied mask dura- 
tion $(40-500 \mathrm{msec})$ to explore the time required to process different types of object information for change detection, once it had been fully encoded. If the configural advantage for 3-D objects that was found by Keane et al. (2003) and Favelle, Palmisano, et al. (2006) was the result of the configural information's being extracted more rapidly or being available earlier in the representation of a visual stimulus than was part shape information, then we would expect to find that changes to this type of information are facilitated at earlier stimulus durations in Experiments 1 and 2. We would also expect change detection decisions based on this type of information to still be facilitated with the shorter mask durations that were examined in Experiment 3. Alternatively, it is possible that configural change detection is better because the configuration of parts is more "salient," or useful, information, in which case, we may find that the configural advantage is not significantly influenced by manipulating the stimulus duration.

\section{EXPERIMENT 1A}

The aim of Experiment 1A was to examine the time course of information extraction for novel 3-D objects. In particular, information regarding the configuration and shape of object parts (in terms of either one part changing shape or two parts switching shape) was investigated. Keane et al. (2003) found a configural advantage using a one-shot change detection task. Because the stimulus durations used in that study were relatively long, subjects should have been able to fully extract all of the object information required to detect these three types of change (the first object stimulus in each trial was shown for $2 \mathrm{sec}$, and the second object stimulus remained on-screen until a response was made). In the present experiment, we used a one-shot change detection task, but examined much shorter stimulus durations (40$500 \mathrm{msec}$ - with a 160 -msec constant masked interstimulus interval [ISI]). The aim was to interrupt information extraction from the object images at different stages of progress. An ISI of $160 \mathrm{msec}$ was selected on the basis of the findings of previous change blindness research with one-shot tasks, which suggested that observers are poor at detecting change whenever displays are separated by an ISI of more than 70-100 msec (Pashler, 1988; Phillips, 1974; Simons, 1996).

Although this will be the first experiment to examine the effects of stimulus duration on the detection of configural and shape changes, it is not the first experiment to examine the effect of stimulus duration on change detection for object properties. For example, Rensink (2000b) investigated orientation and polarity change detection using 2-D stimuli. He manipulated stimulus duration in a "standard" flicker task (with blank fields appearing between successive images), in which participants had to detect the change in a visual search display consisting of 2,6 , or 10 rectangles. Stimulus duration was varied from 80 to $800 \mathrm{msec}$ in this study, with the ISI held constant at 120 msec. Rensink found similar search slopes for detecting changes to the polarity and orientation of the target between 80 and $640 \mathrm{msec}$, which suggested that the processing time for extracting these orientation and polarity properties was approximately constant.

Although Rensink (2000b) found no differences in processing times for orientation and polarity change detection, there is research that suggests an advantage to configuration extraction processing time in change detection. The results of Kimchi $(2000,2003)$ appeared to show that configural properties are used to group line elements (into simple 2-D objects) as early as $40 \mathrm{msec}$. Thus, it is possible that the configural advantage in change detection will also arise early in the processing of complex 3-D objects. Note, however, that the change detection task used in the present study is quite different from the priming tasks used in these previous studies. In addition, Kimchi $(2000,2003)$ referred to configural properties in terms of holistic properties of a group, which is different from how we have operationalized the term. In the present experiment, we compare change detection performance in a one-shot task at five stimulus durations.

\section{Method}

Participants. A total of 53 undergraduate students participated and were tested individually. The participants received course credit for participating.

Materials. The stimuli were rendered images of 3-D novel objects that were similar to those used by Keane et al. (2003). These novel objects had simple configurations. They were constructed from geons (Biederman, 1987) and, as a result, had comparable parts (as opposed to many everyday objects). The aim was to control for any innate or learned preferences for particular part shapes or configurations (e.g., the configuration of features within a face). Each object was composed of a main body with three adjoining parts. The parts attached to the body at three of six possible positions (see Figure 1 for an example). There were 3 "base" objects, each having three configuration, identity, and switch changes made to them, giving a total of 30 different object exemplars used in the present experiment ( 27 changed objects and 3 unchanged objects). Configuration changes always involved one of the three parts changing its location (relative to the body and the two other parts). Switch changes involved two object parts switching positions, with the third part remaining unchanged. Shape changes involved one of the three parts changing shape. All of the objects were photorealistically rendered with the same color and texture. Objects were shown at the same orientation and magnification. They all had a similar size, with the average dimensions of each object being $7.6^{\circ}$ of visual angle wide and $7.4^{\circ}$ of visual angle high. The mask used in Experiment 1 consisted of elements taken from a variety of object images. The entire background of the screen was white (for both image and mask displays).

The experiment was controlled by RSVP software (www.tarrlab .org) running on a Macintosh G4 computer. The presentation timing accuracy of this software was tested using a phototransistor (with a rise/fall time of $6 \mu \mathrm{sec}$ ) that was connected via a circuit board to a Tektronix oscilloscope (TD2-220). The input to the phototransistor was isolated using an opaque tube, so that only the light emitted from a 4-cm-diameter screen region was received. We measured the temporal responding of this phototransistor during an infinite stimulus-mask loop (with an ISI of $10 \mathrm{msec}, 50 \mathrm{msec}$, or $100 \mathrm{msec}$ ). On the basis of these observations, we concluded that the experimental error introduced by RSVP approximated $10 \mathrm{msec}$. The primary cause of this experimental error appeared to 


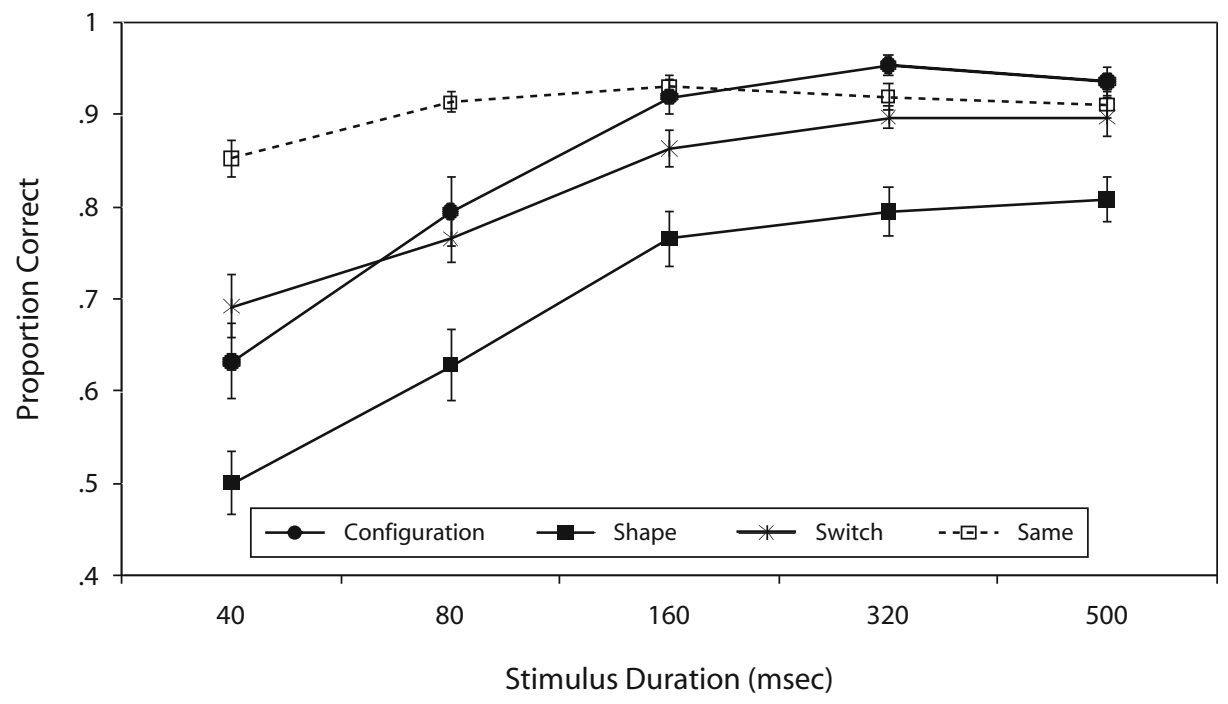

Figure 2. Mean proportion correct of the change detection task in Experiment $1 \mathrm{~A}$ as a function of change type and stimulus duration. Error bars represent standard errors of the means.

be the context switching time of the operating system. This level of experimental error was deemed acceptable, since the minimum stimulus durations examined in the present experiment were $40 \mathrm{msec}$ and $80 \mathrm{msec}$.

Procedure. The participants were first verbally instructed how to complete the task, with emphasis placed on both speed and accuracy in responding. Written instructions on how to complete the task were also provided on the computer screen. After reading the instructions, participants completed four practice trials to familiarize themselves with the task. Stimuli used in the practice trials were different from the stimuli used in the task. Following the practice trials, participants were given a chance to ask any questions they might have had about the procedure before continuing with the experiment.

The experiment consisted of 270 randomly ordered trials. In each of these, the participants viewed sequentially presented pairs of objects on a computer monitor. Each object was randomly placed at a position of 25 pixels in any direction from the center of the screen. Each trial began with a fixation cross appearing for $500 \mathrm{msec}$ at the center of the screen, followed by the first object, which was replaced with a mask appearing on the screen for $160 \mathrm{msec}$, and, finally, a second object that was also replaced with a mask. The mask remained on the screen until a response was made or until the trial timed out after $5,000 \mathrm{msec}$. Both the first and second objects appeared for the same length of time: 40 , $80,160,320$, or $500 \mathrm{msec}$. The next trial began $1,000 \mathrm{msec}$ after the participant made a response or the trial timed out. The second object was either identical to the first, or different in one of three ways: (1) spatial configuration, (2) part shape, or (3) a switching of parts (see Figure 1). Participants were asked to indicate whether the two objects presented to them were same or different by pressing corresponding keys on a keyboard. Half of the trials were same trials, and half were different. The different trials were split equally into the three change type conditions (i.e., 45 trials each), and these were equally split into the five stimulus durations (i.e., 9 trials). Ten self-paced rest periods were interspersed at equal intervals throughout the experimental trials.

\section{Results}

No data were removed due to trial time-outs. Change detection accuracy improved as the stimulus duration increased until $160 \mathrm{msec}$, at which point, performance appeared to plateau; however, same decisions were made with high accuracy across all stimulus durations (see Figure 2). The relative detection performance of the different change types at each of the five stimulus durations was of specific interest to the present study. Thus, planned contrasts between the three change types (configural, switch, and shape) were conducted for each of the 40-, 80-, 160-, 320-, and 500-msec stimulus durations. Two sets of contrasts were used to examine the configural advantage: (1) Shape changes were expected to be detected less accurately than both switch and configural changes, and (2) switch changes were expected to be detected less accurately than configural changes.

At each stimulus duration, shape changes were detected with significantly less accuracy than either configuration or switch changes (all $p \mathrm{~s}<.01$ ). Shape change detection was at chance level $(51 \%)$ at the 40 -msec stimulus duration. Performance was above chance for switch and configuration changes at the 40-msec stimulus duration and for all three change types at longer stimulus durations (all $p \mathrm{~s}<.05$ ). Interestingly, at the 40-msec stimulus duration, switch change detection was more accurate than configuration change detection $(p<.05)$. At the $80-\mathrm{msec}$

Table 1

Planned Contrast Analysis of Accuracy Data in Experiment 1A

\begin{tabular}{lrr}
\hline \multicolumn{1}{c}{ Contrast } & $M S_{\mathrm{e}}$ & \multicolumn{1}{c}{$F$} \\
\hline Configuration vs. switch at $40 \mathrm{msec}$ & .29 & 7.71 \\
Configuration vs. switch at $80 \mathrm{msec}$ & .10 & 4.21 \\
Configuration vs. switch at $160 \mathrm{msec}$ & .11 & 6.34 \\
Configuration vs. switch at $320 \mathrm{msec}$ & .15 & 18.47 \\
Configuration vs. switch at $500 \mathrm{msec}$ & .15 & 8.38 \\
Configuration \& switch vs. shape at $40 \mathrm{msec}$ & 4.90 & 35.43 \\
Configuration \& switch vs. shape at $80 \mathrm{msec}$ & 6.19 & 47.77 \\
Configuration \& switch vs. shape at $160 \mathrm{msec}$ & 4.06 & 47.59 \\
Configuration \& switch vs. shape at $320 \mathrm{msec}$ & 2.67 & 33.74 \\
Configuration \& switch vs. shape at $500 \mathrm{msec}$ & 3.30 & 38.83
\end{tabular}

Note-All $d f \mathrm{~s}=(1,52)$. 


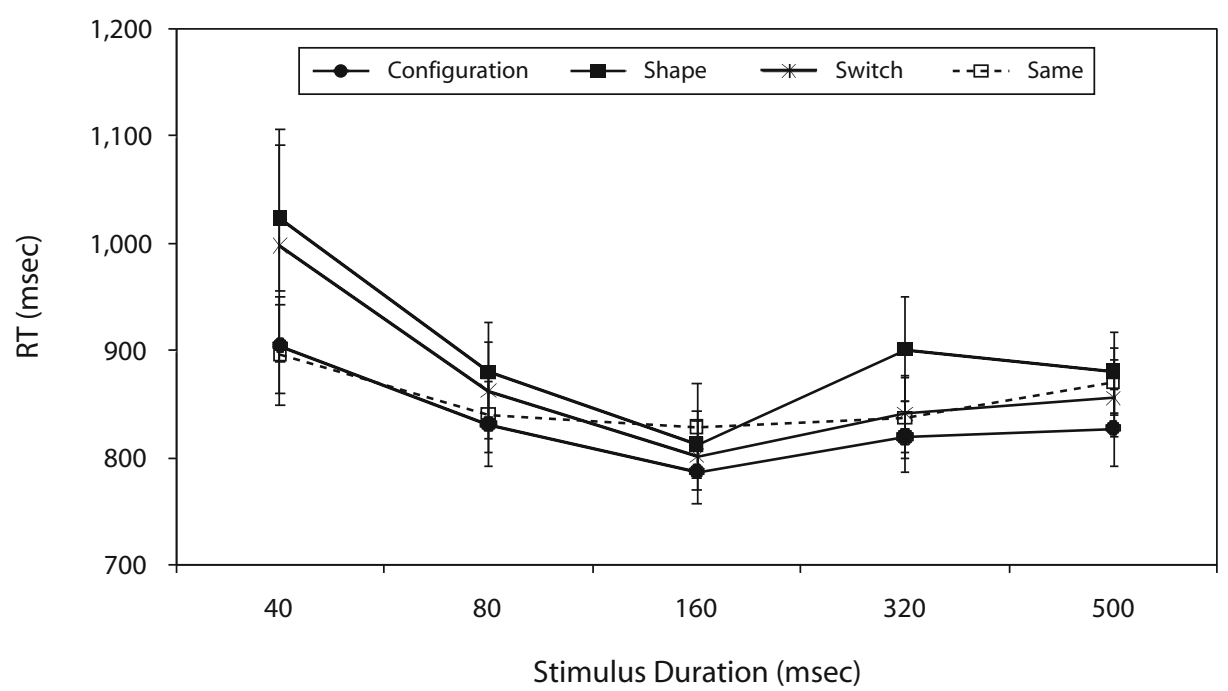

Figure 3. Mean detection time (i.e., RT) on the change detection task in Experiment $1 \mathrm{~A}$ as a function of change type and stimulus duration. Error bars represent standard errors of the means.

stimulus duration, the configural advantage emerged and persisted for 160-, 320-, and 500-msec stimulus duration conditions; that is, configuration changes were detected more accurately than switch changes (all $p \mathrm{~s}<.05$ ). See Table 1 for $F$ values.

A data analysis of RT was conducted on only the accurate responses. The same set of planned contrasts that was used to analyze the accuracy data was applied to the RT data. Despite a general trend to slower RTs for shape change detection and faster RTs for configuration change detection, no significant differences were found between change types at any stimulus duration (all $p \mathrm{~s}>$ .05 ), except that participants were slower to detect shape changes than configural or switch changes at 320 and $500 \mathrm{msec}$ (both $p \mathrm{~s}<.05$; see Figure 3). See Table 2 for $F$ values.

\section{Discussion}

The three change types that were investigated in Experiment $1 \mathrm{~A}$ involve three different types of information about the parts of an object. A configural change involves knowing where the parts are; a shape change involves knowing what parts are in the image; a switch

Table 2

Planned Contrast Analysis of

Reaction Time Data in Experiment 1A

\begin{tabular}{lrr}
\hline \multicolumn{1}{c}{ Contrast } & \multicolumn{1}{c}{$M S_{\mathrm{e}}$} & \multicolumn{1}{c}{$F$} \\
\hline Configuration vs. switch at $40 \mathrm{msec}$ & $290,263.96$ & 2.12 \\
Configuration vs. switch at $80 \mathrm{msec}$ & $25,704.22$ & .83 \\
Configuration vs. switch at $160 \mathrm{msec}$ & $4,924.38$ & .47 \\
Configuration vs. switch at $320 \mathrm{msec}$ & 81.27 & .004 \\
Configuration vs. switch at $500 \mathrm{msec}$ & $6,821.96$ & .45 \\
Configuration \& switch vs. shape at $40 \mathrm{msec}$ & $711,995.03$ & 2.20 \\
Configuration \& switch vs. shape at $80 \mathrm{msec}$ & $106,809.85$ & 1.87 \\
Configuration \& switch vs. shape at $160 \mathrm{msec}$ & $75,740.55$ & 2.49 \\
Configuration \& switch vs. shape at $320 \mathrm{msec}$ & $670,124.18$ & 6.71 \\
Configuration \& switch vs. shape at $500 \mathrm{msec}$ & $245,558.37$ & 4.43 \\
\hline Note-All $d f \mathrm{~s}=(1,52)$ & &
\end{tabular}

Note-All $d f_{\mathrm{s}}=(1,52)$. change involves knowing what parts are where. We found that the participants could detect both configural and switch changes with above-chance accuracy with stimulus durations lasting as little as $40 \mathrm{msec}$, which corresponds with Kimchi's $(2000,2003)$ finding that the configural properties of 2-D objects can be utilized in $40 \mathrm{msec}$. Interestingly, switch changes were more accurately detected than configural changes at $40-\mathrm{msec}$ durations. ${ }^{1}$ This finding is in contrast with current results with longer stimulus durations $(80-500 \mathrm{msec})$, and with previous research using much longer stimulus durations (up to $2,500 \mathrm{msec}$ ), showing that configural changes are always better detected than part switches (Favelle, Hayward, et al., 2006; Favelle, Palmisano, et al., 2006; Keane et al., 2003).

One explanation of these findings is that, in the very early stages of visual information extraction, when given only a $40-\mathrm{msec}$ exposure to a stimulus, change detection may be biased toward some quantitative aspect of the change. In the present study (and also in our previous studies), configural changes to objects produced a greater amount of change (both in terms of the numbers of 2-D silhouette pixels changing and the change in the extent of 3-D surface area) than either switch or shape changes. In our previous studies, which used longer stimulus durations, the numbers of silhouette pixels changing could not account for the configural advantage found for change detection (Favelle, Hayward, et al., 2006; Keane et al., 2003). However, if change detection in the present study was based on this kind of raw quantitative measure for shorter stimulus durations, we should have found a clear configural advantage at $40 \mathrm{msec}$, which we did not.

\section{EXPERIMENT 1B}

In Experiment 1 $\mathrm{A}$ and in experiments in previous studies (Favelle, Hayward, et al., 2006; Favelle, Palmisano, 


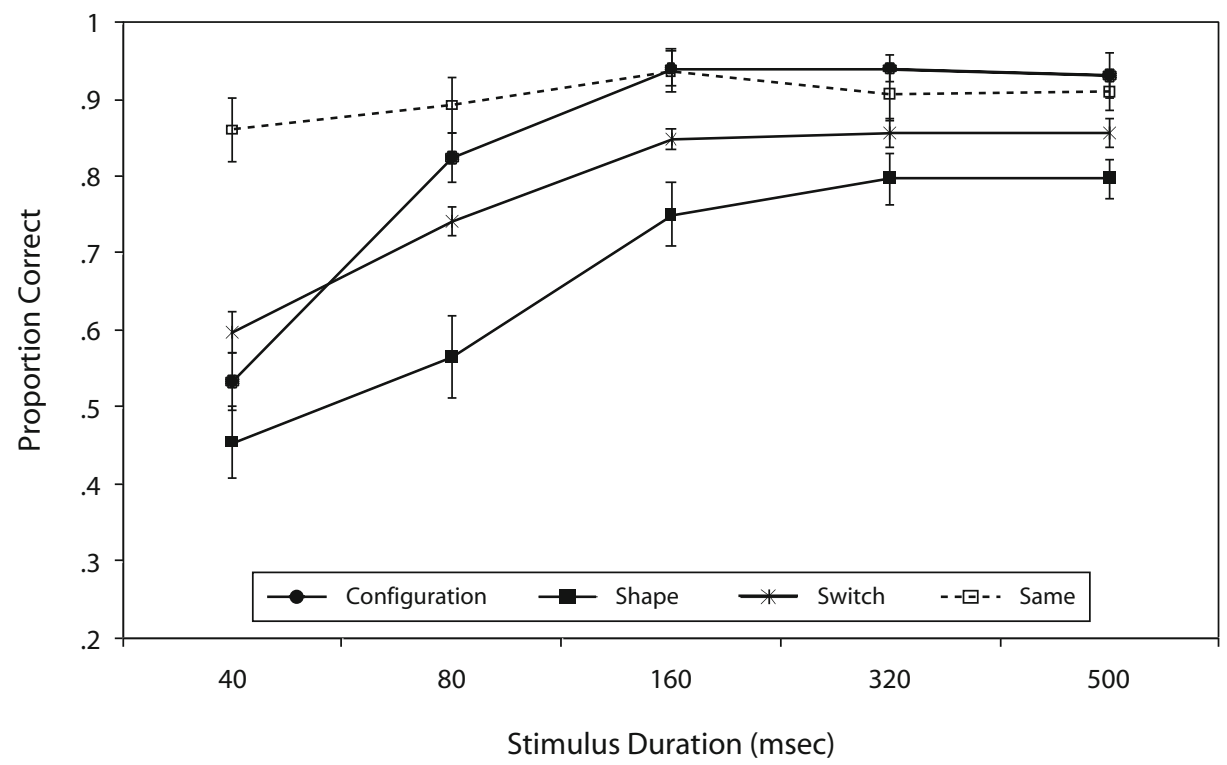

Figure 4. Mean proportion correct on the change detection task in Experiment $1 \mathrm{~B}$ as a function of change type and stimulus duration. Error bars represent standard errors of the means.

et al., 2006; Keane et al., 2003), trial presentation was always randomized so that participants were not able to predict the type of change that they would be exposed to on any given trial. This particular design allowed us to examine the types of information that are spontaneously accessed and utilized in a change detection task. By contrast, Experiment 1B examined change detection performance when the type of change (configuration, switch, or part shape) presented in each block of trials was completely predictable. It was possible that with this type of blocked design, participants might develop strategies to detect each particular change type, and, as a result, performance would improve and differences between the different change types would disappear. ${ }^{2}$

\section{Method}

Participants. Both of the authors and 22 naive students were tested individually, giving a total of 24 participants. Student participants received course credit for participating.

Materials. The materials were the same as those for Experiment $1 \mathrm{~A}$.

Procedure. The procedure was the same as that for Experiment $1 \mathrm{~A}$, except that change type (configuration, shape, and switch) was blocked. The order of blocks was fully counterbalanced between participants. Stimulus duration was randomized within blocks.

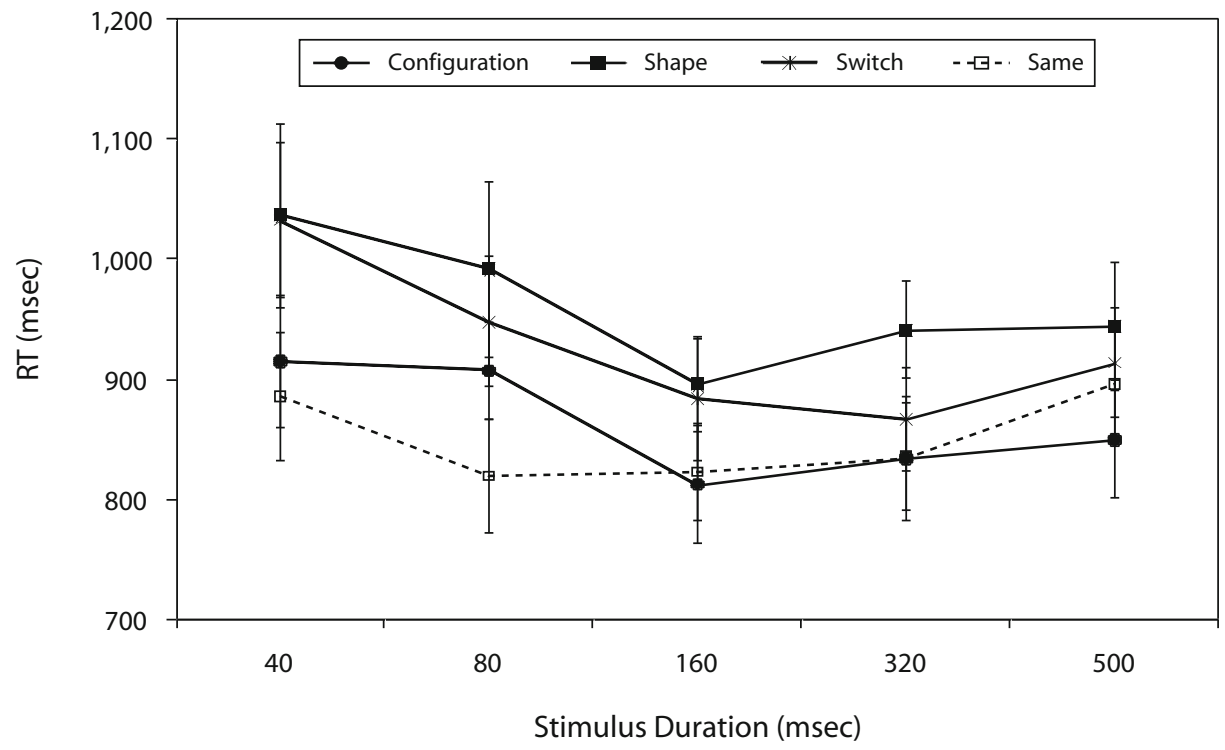

Figure 5. Mean detection time (i.e., RT) on the change detection task in Experiment 1B as a function of change type and stimulus duration. Error bars represent standard errors of the means. 
Table 3

Planned Contrast Analysis of Accuracy Data in Experiment 1B

\begin{tabular}{llr}
\hline \multicolumn{1}{c}{ Contrast } & $M S_{\mathrm{e}}$ & $F$ \\
\hline Configuration vs. switch at $40 \mathrm{msec}$ & 0.10 & 2.00 \\
Configuration vs. switch at $80 \mathrm{msec}$ & 0.17 & 3.03 \\
Configuration vs. switch at $160 \mathrm{msec}$ & 0.21 & 5.69 \\
Configuration vs. switch at $320 \mathrm{msec}$ & 0.17 & 4.28 \\
Configuration vs. switch at $500 \mathrm{msec}$ & 0.13 & 6.24 \\
Configuration \& switch vs. shape at $40 \mathrm{msec}$ & 1.19 & 7.41 \\
Configuration \& switch vs. shape at $80 \mathrm{msec}$ & 4.55 & 18.91 \\
Configuration \& switch vs. shape at $160 \mathrm{msec}$ & 1.98 & 14.63 \\
Configuration \& switch vs. shape at $320 \mathrm{msec}$ & 1.00 & 13.13 \\
Configuration \& switch vs. shape at $500 \mathrm{msec}$ & 0.91 & 10.53 \\
\hline Note-All $d f \mathrm{~s}=(1,23)$ & &
\end{tabular}

Note-All $d f \mathrm{~s}=(1,23)$.

There was a self-paced rest period between each block, and two self-paced rest periods were interspersed at equal intervals within each of the blocks.

\section{Results}

The pattern of results was the same as that for Experiment $1 \mathrm{~A}$ (see Figures 4 and 5). No data were removed because of trial time-outs. Similar analyses were conducted; that is, planned contrasts between the three change types (configural, switch, and shape change) were conducted for each of the 40-, 80-, 160-, 320-, and 500-msec stimulus durations.

At each stimulus duration, shape changes were detected with significantly less accuracy than either configuration or switch changes (all $p \mathrm{~s}<.02$ ). Shape change detection was not different from chance at either the 40 - or 80 -msec stimulus durations $(p>.2)$, nor was configuration change detection different from chance at a 40-msec stimulus duration $(p>4)$. Performance was above chance for all other conditions (all $p \mathrm{~s}<.05$ ). Although the trend was the same as in Experiment 1A, in that switch changes were more accurately detected than configuration changes at $40 \mathrm{msec}$ but not at $80 \mathrm{msec}$, switch change detection was not different from configuration change detection (both $p$ s > .1) at either of these stimulus durations. At a 160 msec stimulus duration, the configural advantage emerged and persisted for 320- and 500-msec stimulus duration conditions; that is, configuration changes were detected more accurately than switch changes (all $p \mathrm{~s}<.05$ ). See Table 3 for $F$ values.

A data analysis of RT was conducted on only the accurate responses. The same set of planned contrasts that was used to analyze the accuracy data was applied to the RT data. A trend similar to that in Experiment 1A of slower RTs for shape change detection and of faster RTs for configuration change detection can be seen in Figure 5, but no statistically significant differences were found between change types at any stimulus duration (all $F \mathrm{~s}<3.7$, $p>.05)$.

\section{Discussion}

Experiment 1B shows the same pattern of results as Experiment 1A, demonstrating that blocking the change type conditions separately has no effect on the time course of the configural advantage. Thus, allowing our participants to select an optimal strategy for each change type did not appear to significantly influence performance. In Experiment 1B, the configural advantage remained absent at shorter stimulus durations and still emerged only at about $160 \mathrm{msec}$, even though participants could focus on the most relevant change detection information (either the parts or the global configuration) for the particular trial. This suggests that the present findings (as well as results from previous studies - e.g., Favelle, Hayward, et al., 2006; Favelle, Palmisano, et al., 2006; Keane et al., 2003) are based on differences in the way that the visual system processes the different object properties of configuration and shape, and not on opportunistic strategy selection.

\section{EXPERIMENT 2}

It has been shown that the magnitude of change can modulate change detection performance (see, e.g., Smilek, Eastwood, \& Merikle, 2000; Williams \& Simons, 2000). Some previous studies have employed post hoc pixelchange analyses to demonstrate that configural properties affect change detection in addition to the magnitude of change (Favelle, Hayward, et al., 2006; Keane et al., 2003). Others have examined this issue directly, by manipulating the number of parts involved in the change as a quantitative measure of the size of change. For example, with long (greater than $1.5 \mathrm{sec}$ ) stimulus durations and changes involving the replacement of 1,2 , or 3 novel object parts, Williams and Simons (2000) found that changes involving more parts were easier to detect than changes involving fewer parts.

In Experiments 1A and 1B, switch changes always involved a two-parts switching location, whereas shape and configural changes always involved only one part. If the object stimulus was not fully encoded during the 40msec exposure, then there would be a greater likelihood that the partial object representation contained one of the two parts involved in a switch change, as compared with the one part involved in a configuration or shape change. Experiment 2 was run to test this idea.

In Experiment 2, we compared detection performance following configuration changes with the following shape changes involving one, two, or three object parts (producing a total of four change type conditions). The one-part configuration changes and one-part shape changes were identical to those in the conditions that were investigated in Experiments 1A and 1B. If it is the case that, with a 40msec exposure, the number of parts changing is a greater determinant of change detection performance than the type of change, then configuration change detection should be worse than the detection of two- or three-part changes. On the basis of the findings of Williams and Simons (2000), we might expect that, regardless of stimulus duration, changes involving three parts should be better detected than changes involving fewer parts. However, if configural information becomes more important for change detection performance as stimulus duration increases, then 


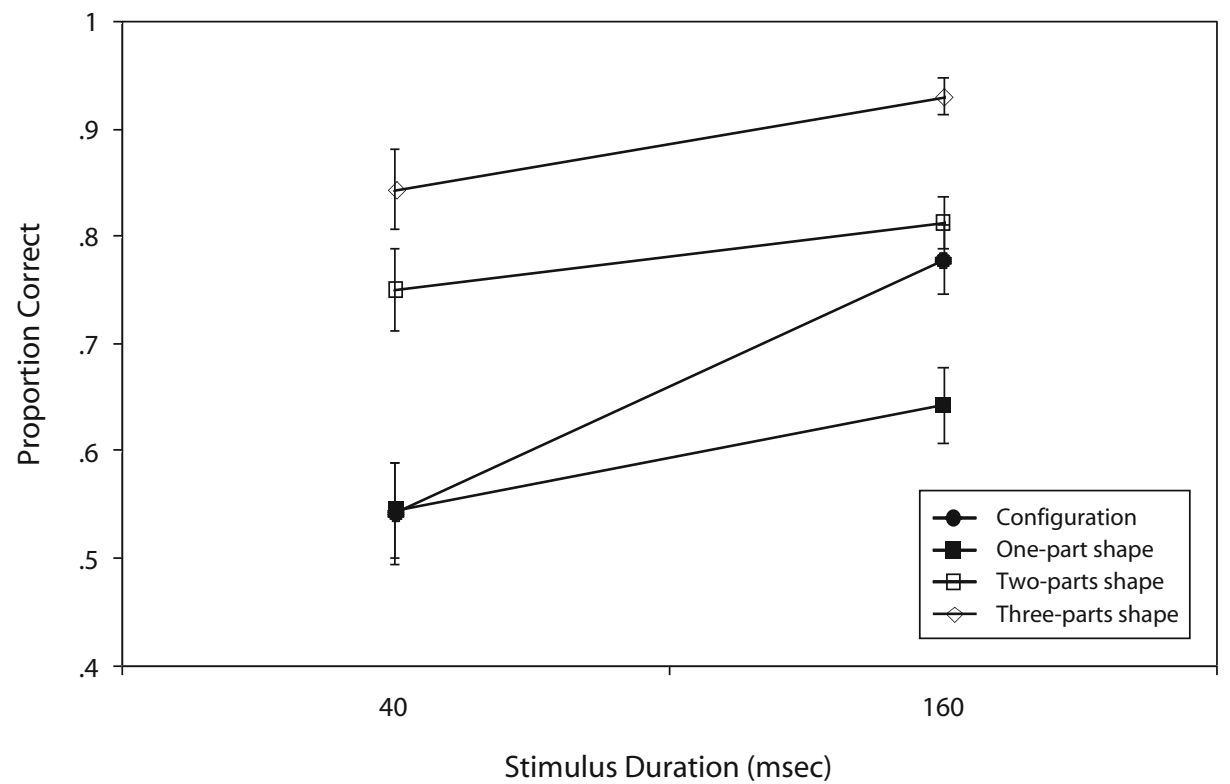

Figure 6. Mean proportion correct on the change detection task in Experiment 2 as a function of change type and stimulus duration. Error bars represent standard errors of the means.

we should see a relative configural advantage emerge at $160 \mathrm{msec}$ (and not at $40 \mathrm{msec}$ ).

\section{Method}

Participants. A total of 16 undergraduate students participated and were tested individually. The participants received course credit for participating.

Materials. The configuration and one-part shape changes were the same as those for Experiments $1 \mathrm{~A}$ and $1 \mathrm{~B}$, with two additional types of object change: (1) two parts changing shape, and (2) three parts changing shape. As for shape changes in Experiments $1 \mathrm{~A}$ and $1 \mathrm{~B}$, shape changes in Experiment 2 saw that the body part remained the same within a trial and that the adjoining parts were replaced.

Procedure. The procedure was the same as that for Experiment $1 \mathrm{~A}$, with the following differences. The experiment consisted of 288 randomly ordered trials. Half of the trials were same trials, and the other half were different. The different trials were split equally into the four change type conditions (i.e., 36 trials each), and these were equally split into the two stimulus durations (i.e., 18 trials). The second object was either identical to the first or different in terms of: (1) one part changing location (i.e., a configural change), (2) one part changing shape, (3) two parts changing shape, or (4) three parts changing shape.

\section{Results}

No data were removed due to trial time-outs. A $4 \times 2$ repeated measures ANOVA including change type (configuration: one-part shape, two-part shape, and three-part shape) and stimulus duration (40 and $160 \mathrm{msec}$ ) was used to analyze the accuracy data (see Figure 6). There was a significant interaction between change type and stimulus duration $\left[F(3,45)=5.75, M S_{\mathrm{e}}=.05, p=.002\right]$. Based on Bonferroni-adjusted pairwise comparisons, this interaction was interpreted as follows: As the stimulus duration increased from 40 to $160 \mathrm{msec}$, there was a large and significant improvement in change detection accuracy for con- figuration changes $\left(M_{\text {config }}=.24, p<.01\right)$, with smaller, only sometimes significant improvements found for shape changes $\left(M_{\text {one-part }}=.10, p<.05 ; M_{\text {two-part }}=.06, p=.09\right.$; $\left.M_{\text {three-part }}=.09, p<.05\right)$. In addition, the 40 -msec stimulus duration configuration changes were detected as accurately as one-part shape changes $(p=1.0)$, and significantly less accurately than any other type of change (all $p \mathrm{~s}<.05$ ). But, at 160-msec stimulus durations, configuration changes were detected significantly more accurately than one-part changes $(p<.05)$ and as accurately as two-part shape changes $(p=1.0)$, but still less accurately for three-part shape changes $(p<.01)$. Overall, changes were detected more accurately with 160 -msec stimulus durations than with 40-msec durations $\left[F(1,15)=12.15, M S_{\mathrm{e}}=.47, p=\right.$ $.003]$, and there was a significant main effect of change type $\left[F(3,45)=62.16, M S_{\mathrm{e}}=.54, p=.000\right]$.

A $4 \times 2$ repeated measures ANOVA including change type (configuration: one-part shape, two-part shape, and three-part shape) and stimulus duration (40 and $160 \mathrm{msec}$ ) was conducted on RT data for accurate responses (see Figure 7$)$. There was a main effect of change type $[F(3,45)=$ $\left.8.1, M S_{\mathrm{e}}=65,623, p=.000\right]$. Bonferroni-adjusted post hoc comparisons showed that three-part shape changes were detected significantly faster than configuration or one-part changes (all $p \mathrm{~s}<.05)$. No significant differences in RT were found between any of the other change types (all $p \mathrm{~s}>$ $.08)$. There was no main effect of stimulus duration on RT $\left[F(1,15)=0.7, M S_{\mathrm{e}}=8,080\right]$. The interaction between stimulus duration and change type also failed to reach significance $\left[F(3,45)=1.1, M S_{\mathrm{e}}=9,075\right]$.

\section{Discussion}

Overall, the results of Experiment 2 show that as the number of parts involved in a change increases, the ac- 


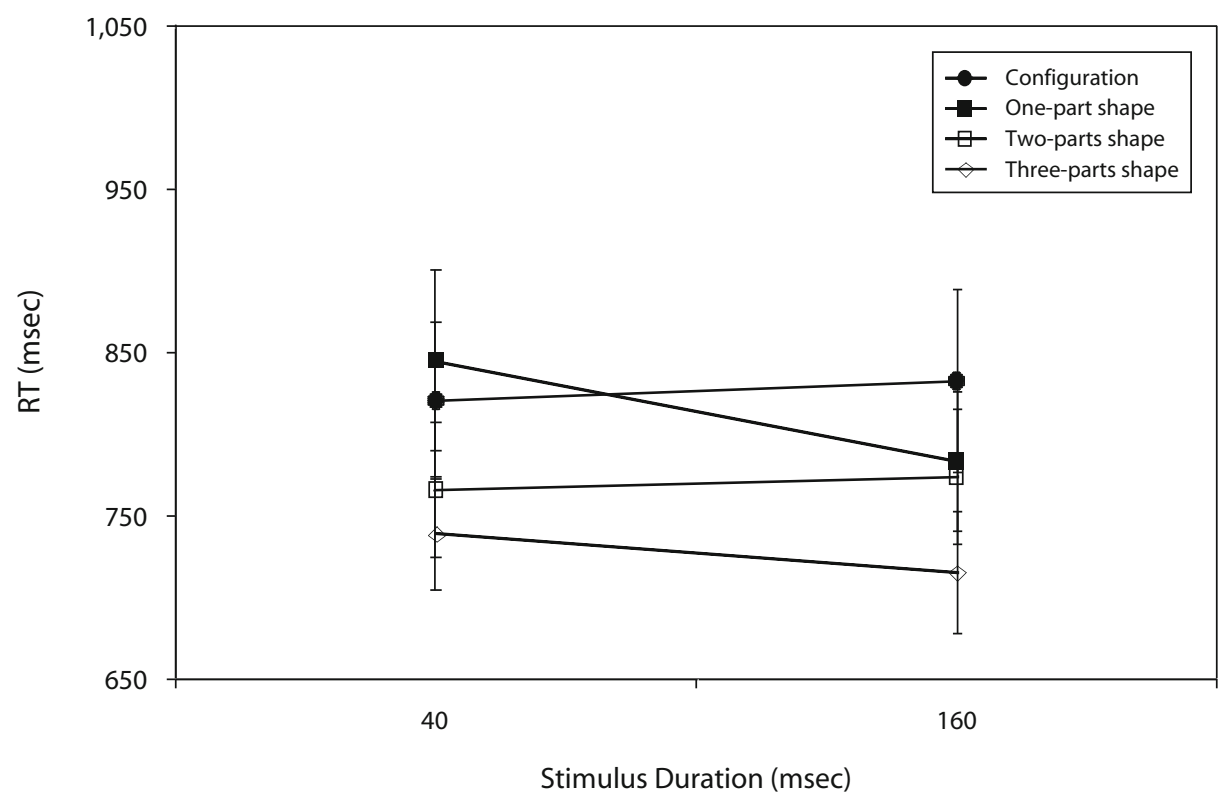

Figure 7. Mean detection time (i.e., RT) on the change detection task in Experiment 2 as a function of change type and stimulus duration. Error bars represent standard errors of the means.

curacy of change detection also increases. This is in line with findings from Williams and Simons (2000). However, stimulus duration interacts with configural change detection. The results of Experiments 1A, 1B, and 2 suggest that change detection with very short stimulus durations is primarily based on the number of parts changing (one quantitative aspect of the change). However, as the stimulus duration increases, configuration change detection improves significantly, whereas shape change does not (or improves only slightly). These results suggest that although shape changes clearly contribute to change detection performance, it is configural information that becomes progressively more important as the object representation develops with time. It appears that we may need at least a $160-\mathrm{msec}$ exposure to a stimulus to reliably extract object layout information and to detect changes to configuration.

An interesting difference in detection performance was observed in Experiment 2. Although configuration changes were more accurately detected than onepart shape changes with 40-msec stimulus durations in Experiments $1 \mathrm{~A}$ and $1 \mathrm{~B}$, detection was not found to be significantly different for these two conditions in Experiment 2 . This discrepancy is likely to have been due to an overall shift in response bias in Experiment 2, given the presence of larger quantitative changes in this experiment (in particular, the very salient three-part shape changes).

\section{EXPERIMENT 3}

Once the different types of information about an object have been extracted from an image, this information must then be retained and further processed in order to allow subsequent change detection. In Experiment 3, we examined the time course of this postexposure information processing by altering the duration of the ISI on change detection for complex objects. We also aimed in this experiment to ascertain the amount of time required to process the different types of object information involved in configural, switch, and one-part shape changes. One explanation of the configural advantage is that postexposure processing of configural information is faster in a change detection task than are other types of information. That is, the time required to determine whether two stimuli have different configural properties is less than that required to determine whether two stimuli differ in terms of their shape properties. The results of Experiments 1A and 1B showed above-chance accuracy for detecting switch changes at a $40-\mathrm{msec}$ stimulus exposure and for detecting configural changes at $40 \mathrm{msec}$ for Experiment 1A (but not for Experiment 1B). However, change detection performance was found to improve and plateau after a stimulus duration of about $160 \mathrm{msec}$ for all change types. The latter finding suggests that all three types of object information can be successfully extracted within this time frame. Since our aim in Experiment 3 was to explore the time course of processing of the different types of object information once they had been extracted, stimulus duration was held constant at $160 \mathrm{msec}$.

Rensink, O'Regan, and Clark (2000, Experiment 2) examined the effect of the duration of the blank fields in a flicker task on the detection of change in central and marginal interest areas of scenes. Their aim was to test whether change blindness was the result of a disruption to the process of consolidating representations necessary for change detection or whether it was due to early-level 


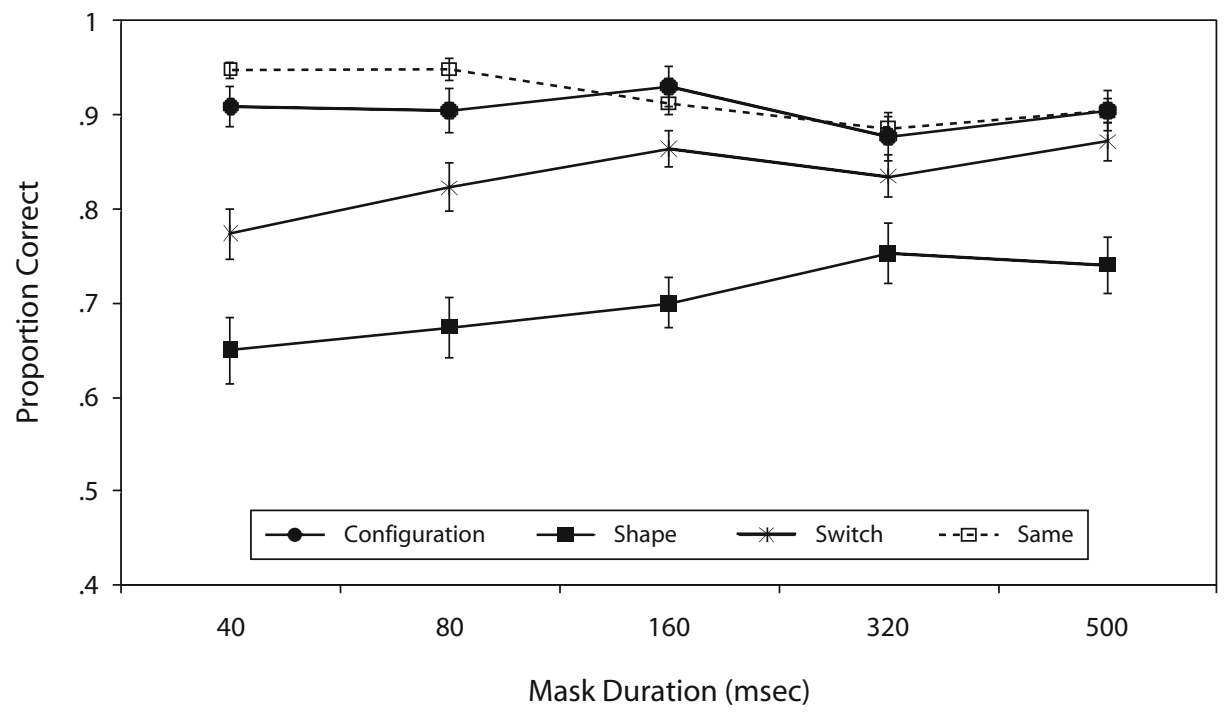

Figure 8. Mean proportion correct on the change detection task in Experiment 3 as a function of change type and mask duration. Error bars represent standard errors of the means.

representations' being volatile. Rensink et al. employed a "standard" flicker task with images presented for $240 \mathrm{msec}$ each, whereas the duration of the interleaved blank field was varied $(40 \mathrm{msec}, 80 \mathrm{msec}, 160 \mathrm{msec}$, or $320 \mathrm{msec}$ ). The pattern of results was complex and did not conclusively support either the volatility or the disruption hypothesis, but, in general, Rensink et al. (Experiment 2) found that longer blank field durations produced longer RTs, regardless of the type of change (i.e., to central or marginal interest areas of the image). Similarly, we expected in Experiment 3 that as the ISI increased, the RT would also increase. As for the effect of ISI duration on the detection of different types of changes, Rensink et al. found no RT differences in detection between central and marginal changes when the ISI was varied. Thus, we might expect that there will be no effect of ISI or mask duration on the RT for detecting the three different change types. However, since we have no evidence relating directly to these three types of changes or to the effects of mask duration on change detection accuracy (as opposed to RT), no firm hypotheses can be made. In a fashion similar to that in Experiments 1A and 1B, the accuracy and RT data analysis examined planned comparisons of the three different change types at each of the five mask durations used in Experiment 3.

\section{Method}

Participants. A total of 27 undergraduate students participated and were tested individually. Subjects received course credit for participating.

Materials. The same materials were used as in Experiments 1A and $1 \mathrm{~B}$.

Procedure and Design. The procedure was identical to that of Experiment 1A with the following exception: Each trial began with a fixation cross appearing for $500 \mathrm{msec}$ at the center of the screen, followed by the first object for $160 \mathrm{msec}$, which was then replaced by a mask of variable duration $(40,80,160,320$, or $500 \mathrm{msec})$, and then by a second object for $160 \mathrm{msec}$, which was finally replaced with a mask that remained on the screen until either a response was made or the trial timed out (after 5,000 msec). The next trial began $1,000 \mathrm{msec}$ after the subject made a response ("same" or "different") or the trial timed out.

\section{Results}

Data from only one trial were removed from analysis due to a time-out. Looking at Figure 8, it appeared that mask duration had little effect on detection accuracy in the same and configural change conditions, yet both switch and one-part shape change detection improved as mask duration increased. Planned linear contrasts showed that detection accuracy did not increase linearly with increasing mask duration within the configuration change condition $[F(1,26)=.41, p=.53]$. However, detection accuracy did increase linearly with increasing mask duration within both the switch and the one-part shape change conditions $[F(1,26)=10.4, p<.05$, and $F(1,26)=5.92, p<.05$, respectively $].$ We conducted the same set of planned contrasts as in the analysis of data in Experiment 1 on the detection accuracy data. The

Table 4

Planned Contrast Analysis of Accuracy Data in Experiment 3

\begin{tabular}{lrr}
\hline \multicolumn{1}{c}{ Contrast } & $M S_{\mathrm{e}}$ & \multicolumn{1}{c}{$F$} \\
\hline Configuration vs. switch at $40 \mathrm{msec}$ & .50 & 16.73 \\
Configuration vs. switch at $80 \mathrm{msec}$ & .18 & 10.36 \\
Configuration vs. switch at $160 \mathrm{msec}$ & .12 & 5.54 \\
Configuration vs. switch at $320 \mathrm{msec}$ & .05 & 1.99 \\
Configuration vs. switch at $500 \mathrm{msec}$ & .03 & .91 \\
Configuration \& switch vs. shape at $40 \mathrm{msec}$ & 3.96 & 19.52 \\
Configuration \& switch vs. shape at $80 \mathrm{msec}$ & 3.87 & 33.06 \\
Configuration \& switch vs. shape at $160 \mathrm{msec}$ & 4.21 & 48.06 \\
Configuration \& switch vs. shape at $320 \mathrm{msec}$ & 1.14 & 9.43 \\
Configuration \& switch vs. shape at $500 \mathrm{msec}$ & 2.37 & 26.84 \\
\hline
\end{tabular}

Note-All $d f \mathrm{~s}=(1,26)$ 


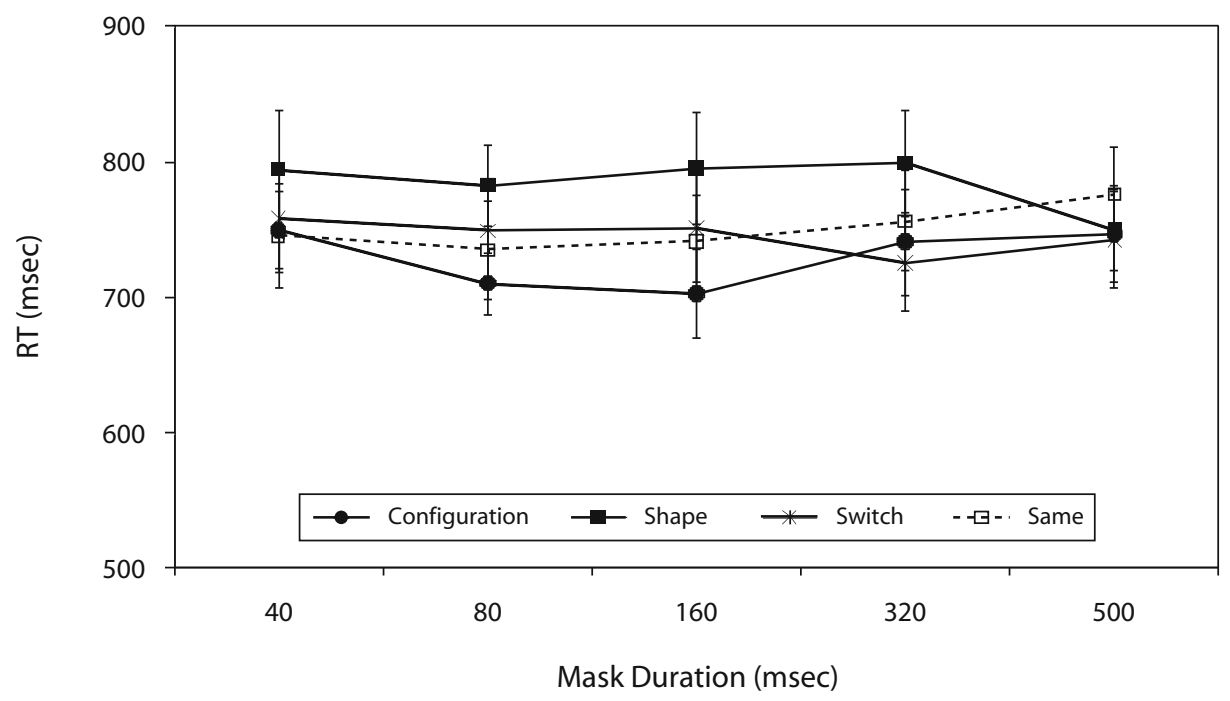

Figure 9. Mean detection time (i.e., RT) on the change detection task in Experiment 3 as a function of change type and mask duration. Error bars represent standard errors of the means.

results showed that across each of the mask durations $(40,80,160,320$, and $500 \mathrm{msec})$, the detection accuracy for one-part shape changes was significantly worse than that for either switch or configuration changes (all $p$ s $<$ $.01)$. Configuration change detection was also significantly more accurate than switch changes at $40-, 80-$, and 160 -msec mask durations (all $p$ s $<.05$ ). However, there was no significant difference between configuration and switch changes at 320- or 500-msec mask durations (both $p$ s $>.05$ ). See Table 4 for $F$ values.

Data analysis of RT was conducted on only the accurate responses. Contrary to expectations, the planned linear contrast analysis showed that the RT did not increase linearly with increasing mask duration within any of the change conditions [configuration, $F(1,26)=$ $.21, p=.65$; switch, $F(1,26)=.93, p=.34$; and shape, $F(1,26)=1.5, p=.23]$. The set of planned contrasts corresponding to those in Experiment 1 showed that configural changes were detected more quickly than switch changes at 160 -msec mask durations $(p<.05)$, and that one-part shape changes were detected more slowly than configural and switch changes at 80-, 160-, and

Table 5

Planned Contrast Analysis of

Reaction Time Data in Experiment 3

\begin{tabular}{lrr}
\hline \multicolumn{1}{c}{ Contrast } & \multicolumn{1}{c}{$M S_{\mathrm{e}}$} & \multicolumn{1}{c}{$F$} \\
\hline Configuration vs. switch at $40 \mathrm{msec}$ & $1,965.60$ & .13 \\
Configuration vs. switch at $80 \mathrm{msec}$ & $40,871.61$ & 2.48 \\
Configuration vs. switch at $160 \mathrm{msec}$ & $64,358.46$ & 5.48 \\
Configuration vs. switch at $320 \mathrm{msec}$ & $6,291.59$ & .18 \\
Configuration vs. switch at $500 \mathrm{msec}$ & 483.60 & .03 \\
Configuration \& switch vs. shape at $40 \mathrm{msec}$ & $177,930.17$ & 2.68 \\
Configuration \& switch vs. shape at $80 \mathrm{msec}$ & $304,680.25$ & 6.14 \\
Configuration \& switch vs. shape at $160 \mathrm{msec}$ & $506,670.41$ & 9.51 \\
Configuration \& switch vs. shape at $320 \mathrm{msec}$ & $467,038.72$ & 4.62 \\
Configuration \& switch vs. shape at $500 \mathrm{msec}$ & $3,253.93$ & .07 \\
\hline
\end{tabular}

Note-All $d f \mathrm{~s}=(1,26)$. 320-msec mask durations $(p<.05)$ (see Figure 9). All remaining change type differences in RT (i.e., at other mask durations) failed to reach significance (all $p \mathrm{~s}>$ $.05)$. See Table 5 for $F$ values.

\section{Discussion}

The results showed that there were differences in the speed with which fully encoded object properties (configuration and shape of parts) could be used to successfully detect change. Configural information for change detection was retained and used accurately across all of the mask durations tested. However, switching part shapes and one-part shape replacement change detection accuracy reached maximal performance only at around 160and 320-msec mask durations, respectively. This pattern of results suggests that the postexposure processing time required to accurately detect a switch change or a shape change was four to eight times longer than that to detect a configuration change. We failed to find an increase in RT with increasing ISI. This might reflect a difference in processing scenes (Rensink et al., 2000) as opposed to the single 3-D objects examined in the present study. Alternatively, the failure to find an increase in RT could be due to our experiments using a mask during the ISI, whereas Rensink et al. used a blank field.

\section{GENERAL DISCUSSION}

The goal of the present study was to determine the time course of extracting and processing configural and component shape properties in 3-D objects using a one-shot change detection task. In Experiment 1A, we manipulated stimulus duration $(40-500 \mathrm{msec})$ in order to determine the stimulus exposure required to extract configural, part shape, and arrangement (i.e., switching parts) information. Although a detection advantage was found for switch changes over configuration and 
part shape changes with $40-\mathrm{msec}$ stimulus durations, a configural advantage emerged at 80 -msec stimulus durations and persisted for longer stimulus durations. Interestingly, detection performance for all three change types was found to plateau from the same stimulus duration $(160 \mathrm{msec})$. In Experiment 1B, we showed that the same pattern of results was obtained when change conditions were blocked (allowing participants to develop different strategies for different change types). This suggests that change detection performance was determined by the nature of the visual information being processed and not by the participants' adopting possible compromise strategies. In Experiment 2, we showed that at 40msec stimulus durations, the number of parts changing was a better predictor of detection performance than the qualitative nature of the change. But, at longer stimulus durations, the overall configuration of the object's parts appeared to play an increasingly important role in object processing and change detection performance. Finally, in Experiment 3, we manipulated ISI mask duration $(40-500 \mathrm{msec})$ to determine the time required to process these different types of object information for change detection, once the information had been fully encoded (i.e., stimulus duration was held constant at $160 \mathrm{msec}$ ). Although configural change detection was unaffected by mask duration manipulations (performance appeared to be at ceiling levels with ISIs as short as $40 \mathrm{msec}$ ), mask durations of approximately $160-320 \mathrm{msec}$ appeared necessary for switch and shape changes to reach peak performance accuracy.

In general, we found the expected configural advantage over switch and shape changes-at least when these different change types were similar in terms of the number of parts changing ${ }^{3}$ (Favelle, Hayward, et al., 2006; Favelle, Palmisano, et al., 2006; Keane et al., 2003). However, this configural advantage emerged only at stimulus durations of $160 \mathrm{msec}$ or longer. With shorter, 40-msec stimulus durations, we instead found a switch advantage over configuration and one-part shape changes in Experiments 1A and 1B, and no significant difference between configuration and one-part shape changes in Experiment 2. Thus, we propose that $40 \mathrm{msec}$ is too short an exposure to create a complete representation of "what" and "where" all of the parts are of the 3-D objects used in this study. Twice as many parts were involved in a switch change as in either a one-part configuration change or a one-part shape change. If one assumes that only a partial object representation can be created with a 40-msec stimulus exposure, then the probability of such a representation containing one of the two parts involved in a switch change will be greater than the probability of its containing the one part involved in a configuration or shape change. Thus, it seems that at very short stimulus exposures, the number of parts or the proportion of the 3-D object involved in a change is more important for successful change detection than the type of change. This appears to be at odds with findings of configural property dominance over components in early vision (see, e.g., [Kimchi's] 2000, 2003 research on 2-D objects); however, in Experiment 1A, configural change detection was both significantly above chance and more accurate than shape change detection at a 40msec stimulus duration.

The results of Experiment 3 revealed significant differences in the length of postexposure processing required for accurate configural, part shape, and switch change detection. Configural change detection was found to be highly accurate and largely unaffected by the duration of the ISI mask, suggesting that very little postexposure processing was required to successfully detect a configural change. Conversely, both switch and shape change detection were found to improve with ISI durations up to 160 and $320 \mathrm{msec}$, respectively. These findings indicate that, once extracted, configural information is utilized much faster than either shape or location information (at least when generating a change detection decision). This is in line with research by Kimchi and Bloch (1998), who found that when both configural and component properties were available in discrimination and classification tasks, configural properties dominated performance.

Taken together, the present results show that (1) the amount of change (parts or pixels) determines 3-D object change detection performance at very brief stimulus exposures, and (2) some 3-D configural information can be extracted with short stimulus exposure, but a complete representation of 3-D configural information takes time. This is in line with a system whose primary goal is object identification and recognition, in which the earliest available information is used to determine the lowlevel properties of an object (e.g., size, surface parsing, segmenting of regions on the basis of shared texture) necessary for the development of higher level properties, such as configuration.

As was mentioned previously, speed is just one aspect of efficient information processing. We have shown in Experiment 3 that once extracted, configural or structural information appears to be processed more quickly than local shape information in 3-D objects. This finding has implications for theories of object recognition in that it suggests that structural and featural information is processed separately, and that the representation of structural information may have some priority. Theories in which object recognition is achieved by template matching or with representations that do not individuate (parts or structural) features (see, e.g., Ullman, 1989) cannot account for the present findings. Likewise, pure featural accounts that do not explicitly encode the position or location of a set of localized features (e.g., Mel, 1997) will also fail to account for these findings. There are, however, a number of theories that ascribe a distinct role to the representation of structural information, including structural description theory (Marr \& Nishihara, 1978), recognition-by-components and its variants (Biederman, 1987; Biederman \& Gerhardstein, 1993; Hummel \& Biederman, 1992), and chorus of fragments (Edelman \& Intrator, 2000, 2003). Note that the present results speak to the question of whether structural relations and features are encoded separately and not to the 
specific nature of the representations themselves (see also Barenholtz \& Tarr, 2006).

In the present study, the stimuli were all depictions of 3-D objects. This raises the question of whether the same results would be expected for similar 2-D objects (e.g., silhouette versions of our objects)? There are some important differences between the findings of the present study on 3-D objects and those of earlier studies using 2-D objects (although, as was noted earlier, these could be due to methodological differences). Thus, we would not be surprised if 3-D structure plays an important role in object change detection. However, since we did not directly test 2-D versus 3-D structure, addressing this question is beyond the scope of the present study. It should clearly be the focus of future research.

In conclusion, the present results were generally consistent with previous research demonstrating a configural advantage for 3-D object change detection (Favelle, Hayward, et al., 2006; Favelle, Palmisano, et al., 2006; Keane et al., 2003). However, our experiments have shown that stimulus duration plays a critical role in the emergence of this configural advantage. With short stimulus durations $(40 \mathrm{msec})$, change detection was best predicted by the magnitude of the change (i.e., not by the type of change, but rather by how many parts were involved in the change). Superior configural change detection was found to emerge only when observers were given at least 160 -msec stimulus exposure, which thus provides a rough estimate of the minimum amount of time required to extract useful configural information from 3-D objects. Interestingly, although this configural advantage persisted for longer stimulus durations, detection performance plateaued for all three types of change (configural, switch, and shape), with stimulus durations around $160 \mathrm{msec}$. Our results suggest that much of the configural advantage for 3-D objects arises during postexposure processing. Although manipulations of the postexposure processing time had no significant effect on configural change detection (highly accurate performance was evident at all mask durations), peak switch and shape change detection required a minimum of $160-$ or $320-\mathrm{msec}$ postexposure processing, respectively. Taken together, these findings are consistent with models of visual object processing in which configural properties dominate performance via their speedier processing and more effective utilization.

\section{AUTHOR NOTE}

The authors thank Will Hayward, Ron Rensink, and two anonymous reviewers for their helpful comments and suggestions in relation to earlier drafts of the article. Correspondence concerning this article should be addressed to S. Favelle, School of Psychology, University of Wollongong, Northfields Ave., Wollongong 2522, NSW, Australia (e-mail: simone_favelle@uow.edu.au).

\section{REFERENCES}

Aginsky, V., \& TARr, M. J. (2000). How are different properties of a scene encoded in visual memory? Visual Cognition, 7, 147-162.

Barenholtz, E., Cohen, E., Feldman, J., \& Singh, M. (2003). De- tection of change in shape: An advantage for concavities. Cognition, 89, 1-9.

Barenholtz, E., \& TARR, M. J. (2006). Reconsidering the role of structure in vision. In B. H. Ross (Series Ed.) \& A. B. Markman (Vol. Ed.), Categories in use: The psychology of learning and motivation (Vol. 47, pp. 157-180). San Diego: Academic Press.

Biederman, I. (1987). Recognition-by-components: A theory of human vision understanding. Psychological Review, 94, 115-145.

Biederman, I., \& Gerhardstein, P. C. (1993). Recognizing depthrotated objects: Evidence and conditions for three-dimensional viewpoint invariance. Journal of Experimental Psychology: Human Perception \& Performance, 19, 1162-1182.

BLum, H. (1973). Biological shape and visual science. I. Journal of Theoretical Biology, 38, 205-287.

Edelman, S., \& Intrator, N. (2000). (Coarse coding of shape fragments $)+($ retinotopy $) \approx$ representation of structure. Spatial Vision, 13, 255-264.

Edelman, S., \& Intrator, N. (2003). Towards structural systematicity in distributed, statically bound visual representations. Cognitive Science, 27, 73-110.

Favelle, S. K., Hayward, W. G., Burke, D., \& Palmisano, S. (2006). The configural advantage in object change detection persists across depth rotation. Perception \& Psychophysics, 68, 1254-1263.

Favelle, S. K., Palmisano, S., Burke, D., \& Hayward, W. G. (2006). The role of attention in processing configural and shape information in 3D novel objects. Visual Cognition, 13, 623-642.

Hochberg, J. E. (1968). Perception. Englewood Cliffs, NJ: Prentice Hall.

Hollingworth, A., \& Henderson, J. M. (2002). Accurate visual memory for previously attended objects in natural scenes. Journal of Experimental Psychology: Human Perception \& Performance, 28, 113-136.

Hummel, J. E., \& Biederman, I. (1992). Dynamic binding in a neural network for shape recognition. Psychological Review, 99, 480-517.

Keane, S. K., Hayward, W. G., \& Burke, D. (2003). Detection of three types of changes to novel objects. Visual Cognition, 10, 101-127.

KIмCHI, R. (2000). The perceptual organization of visual objects: A microgenetic analysis. Vision Research, 40, 1333-1347.

KImCHI, R. (2003). Microgenetic analysis of visual perceptual organization. In R. Kimchi, M. Behrmann, \& C. R. Olson (Eds.), Perceptual organization in vision: Behavioral and neurological perspectives. Mahwah, NJ: Erlbaum.

Kimchi, R., \& BLOCH, B. (1998). Dominance of configural properties in visual form perception. Psychonomic Bulletin \& Review, 5, 135-139.

Kimia, B. B., Tannenbaum, A. R., \& Zucker, S. W. (1995). Shapes, shocks, and deformations I: The components of two-dimensional shape and the reaction-diffusion space. International Journal of Computer Vision, 15, 189-224.

MARR, D., \& Nishinara, H. K. (1978). Representation and recognition of the spatial organization of three-dimensional shapes. Proceedings of the Royal Society B, 200, 269-294.

MeL, B. (1997). SEEMORE: Combining color, shape and texture histogramming in a neurally inspired approach to visual object recognition. Neural Computation, 9, 777-804.

PASHLER, H. (1988). Familiarity and visual change detection. Perception \& Psychophysics, 44, 369-378.

Phillips, W. A. (1974). On the distinction between sensory storage and short-term visual memory. Perception \& Psychophysics, 16, 283-290.

Pomerantz, J. R. (1983). Global and local precedence: Selective attention in form and motion perception. Journal of Experimental Psychology: General, 112, 516-540.

Rensink, R. A. (2000a). The dynamic representation of scenes. Visual Cognition: Special Issue on Change Detection \& Visual Memory, 7, 17-42.

Rensink, R. A. (2000b). Visual search for change: A probe into the nature of attentional processing. Visual Cognition, 7, 345-376.

Rensink, R. A., O'Regan, J. K., \& Clark, J. J. (2000). On the failure to detect changes in scenes across brief interruptions. Visual Cognition, 7, 127-145. 
Simons, D. J. (1996). In sight, out of mind: When object representations fail. Psychological Science, 7, 301-305.

Simons, D. J. (2000). Current approaches to change blindness. Visual Cognition, 7, 1-15.

Simons, D. J., \& Levin, D. (1997). Change blindness. Trends in Cognitive Sciences, 1, 261-267.

Simons, D. J., \& Rensink, R. A. (2005). Change blindness: Past, present, and future. Trends in Cognitive Sciences, 9, 16-20.

Smilek, D., Eastwood, J. D., \& Merikle, P. M. (2000). Does unattended information facilitate change detection? Journal of Experimental Psychology: Human Perception \& Performance, 26, 480-487.

UlLman, S. (1989). Aligning pictorial descriptions: An approach to object recognition. Cognition, 32, 193-254.

Williams, P., \& Simons, D. J. (2000). Detecting changes in novel 3D objects: Effects of change magnitude, spatiotemporal continuity, and stimulus familiarity. Visual Cognition, 7, 297-322.

\section{NOTES}

1. Looking at Figures 2 and 3, there appears to be a speed-accuracy trade-off between switch and configuration change at a 40-msec stimulus duration; however, participants did not take significantly longer to respond to switch changes than to configural changes $(p=.1)$.

2. Thanks to a reviewer for this suggestion.

3. It should be noted that we did actually find the best detection performance, in terms of both accuracy and RT, for the largest object changes (i.e., two- and three-part shape changes) across all stimulus durations, as was expected from Williams and Simons (2000).

(Manuscript received June 29, 2009;

revision accepted for publication January 16, 2010.) 